\title{
Pyomyositis, a case report and review of the literature
}

\begin{abstract}
Pyomyositis is an infection of the skeletal muscle most commonly caused by Staphylococcus aureus. Most cases have been reported in tropical countries; however, there has been an increasing amount of cases documented in immunocompromised children in North America. It is hypothesized that pyomyositis may actually be under diagnosed in no tropical locations and thus, practitioners should have a higher index of suspicion when a patient presents with vague symptoms of fever and muscle pain. We report the case of a 7 month old African American male who presented with vague signs of left calf swelling, pain, and fever. These symptoms persisted and developed over about six days before being admitted to the hospital. Upon admission, his left calf was noted to be diffusely swollen and warm to touch with increased firmness and tightness in the posterior upper left calf. He was also found to have a leukocytosis of 24.1, elevated erythrocyte sedimentation rate and c-reactive protein of 84 and 3.646 , and an ultrasound which revealed an abscess measuring $1.9 \times 2.7 \times 3.7 \mathrm{~cm}$ involving the upper left calf musculature. This case is an unusual presentation of a patient significantly younger than the mean age of 5 years old in children diagnosed with pyomyositis in the United States. Additionally, he had no official diagnosis of immunodeficiency or obvious trauma to the area. Although his original symptoms describe stage 1 pyomyositis, he rapidly progressed to stage 2 with abscess formation in less than one week and thus was managed with surgical incision and drainage of the area. Wound cultures collected at the time of the surgery revealed methicillin-resistant Staphylococcus aureus which guided our antibiotic therapy. He was sent home on oral clindamycin for a total antibiotic course of 14 days.
\end{abstract}

Keywords: pyomyositis, Staphylococcus aureus, penrose drain, Eagle effect, abscess, immunodeficiency, antibiotics, Vancomycin, vaccines, clindamycin, aspiration, calf muscles
Volume 6 Issue 4 - 2018

\author{
Vanessa Sui,' Richard A Giovane, ${ }^{2}$ Larab \\ Ahmed, ${ }^{2}$ Heather Taylor ${ }^{3}$ \\ 'University of Birmingham, School of Medicine, USA \\ 2Departmentof Family Medicine, University of Alabama, USA \\ ${ }^{3}$ Department of Pediatrics, University of Alabama, USA
}

Correspondence: Richard Giovane, Resident Physician at the University of Alabama, $8505^{\text {th }}$ ave, Peter Bryce Blvd, USA, Tel 91799327 94, Email richardgiovane357@gmail.com

Received: July 10, 2018 | Published: July 17, 2018

\section{Introduction}

Pyomyositis is an infection most predominantly in tropical countries that affects the skeletal muscle and often coincides with abscess formation. ${ }^{1}$ Both children and adults may be affected, but in the pediatric population, most patients are around 5 years old and have predisposing risk factors such as trauma, immunodeficiencies, or malnutrition. It most commonly affects large muscle groups in the hips and thighs such as the quadriceps, iliopsoas, and gluteal muscles. ${ }^{2}$ Causes of pyomyositis include non-group a streptococci, pneumococci, E. coli, and Staphylococcus aureus which contributes to up to $70 \%$ of cases. However, methicillin-resistant Staphylococcus aureus has become an important etiology of pyomyositis in recent years due to an increasing amount of antibiotic resistance and pediatric cases in the nosocomial setting, especially in premature infants. ${ }^{3}$

The infection spreads hematogenously and can be rapidly progressive from localized in the muscle to abscess formation and sepsis within days. Treatment is often delayed due to nonspecific symptoms and recent literature reports that the average time from admission to diagnosis is around 7-10 days $^{4-5}$ additionally, the differential diagnosis for vague musculoskeletal pain is extensive which also contributes to a delay in treatment and diagnosis.

\section{Case presentation}

We report the case of a 7 month old African American male with a past medical history of preterm birth at 31 weeks who presented as a direct admit from clinic due to left calf swelling and pain with associated fever. His mother states that about 5 days prior, his early intervention therapist noticed that he was avoiding putting weight on his left leg. She also noticed some mild swelling that she believed involved both his upper and lower left leg. She thought that he may have injured his leg kicking or playing and therefore, provided him with some ibuprofen and expected the pain and swelling to resolve. The next day, she believed that the swelling had worsened and also become more localized to his upper left calf. Therefore, she called her local emergency department for evaluation. At the emergency department, $\mathrm{x}$-rays were performed which showed no fractures or acute abnormalities. She was advised that it was most likely a muscle sprain and to provide him with ibuprofen with follow-up at his pediatrician if there was no improvement. He presented to the clinic the next day with no improvement and with fever of 101.1 which was his first documented fever during this course for which he was admitted to the hospital. Mom reports that the patient refuses to extend his left knee or put any weight on his left leg, but is still moving the leg while sitting and laying down. He becomes agitated when others move his left leg, but is otherwise acting like himself. She does not think that the skin over the swelling has been erythematous and she denies any history of known trauma to the area including break in the skin or bug bites. He has not had cough, congestion, vomiting, and change in number of voices or rash.

He received his 6 month vaccines which included DTaP, Hep $\mathrm{B}$, HIB, pneumococcal, polio, and rotavirus 2 weeks prior to this 
presentation which caused some erythema and mild swelling at the injection site of his left lateral thigh, but his mother confirms that these symptoms resolved within 2 days.

Our patient was born at 31.1 to a 25 -year old G3P2103 via repeat lower transverse cesarean section. He weighed 1825 grams at birth and his APGARs were 9/9 at 1minute and 5minutes, respectively. His mother's pregnancy was complicated by preterm premature rupture of membranes and preterm labor. She was GBS positive but received adequate ampicillin treatment during her delivery. He remained in the NICU for about 3 months due to respiratory distress syndrome, anemia of prematurity, low birth weight, nutritional support, bronchopulmonary dysplasia, and a $1.5 \times 1.4 \times 2 \mathrm{~cm}$ selfresolving submandibular neck mass that was determined to be likely due to lymphadenopathy and thus followed outpatient. His newborn screenings were all within normal limits and he is followed by an interventional therapist due to his prematurity and developmental delay. He was diagnosed with laryngomalacia at around the age of 4 months old. His only surgery includes a circumcision and his immunizations are up-to-date, although he did not receive a flu vaccine during the previous winter. He has no known allergies and takes no medications at this time. Additional information about this patient includes no family history of autoimmune disease, immunodeficiency, joint disease, or malignancy. His first-degree relatives are all relatively healthy. Our patient lives in a home with his mother, maternal grandmother, and two older sisters. There are no pets or firearms at home. He is not exposed to passive smoke. He is currently not in daycare and he and his siblings are taken care of by his mother full-time - none are at school-age.

On presentation, our patient had a low-grade temperature of 100.2, pulse of 140 , and respiratory rate of 40 . He weighed about 13.25pounds which was less than $5^{\text {th }}$ percentile for his age group but stable since birth. His height was about 26.25 inches which was in the $10^{\text {th }}$ percentile for his age group and had increased from 5 th percentile at birth. He was in no apparent distress and was alert and active during our exam. His left lower extremity was diffusely swollen with increased firmness and tightness in the posterior upper left calf. Upon palpation, our patient cried and was in obvious pain. There was no erythema, open wounds, rashes, petechiae, or obvious areas of trauma to the left lower extremity. It was slightly warm to touch compared to right lower extremity but there were no obvious areas of induration or fluctuance. He resisted extension and flexion of the left knee joint and extension of the left hip joint. He also kept his lower left extremity in a position with his knees and hips flexed.

\section{Tests and imaging}

Upon admission, lab work was performed to further investigate the source of the patient's symptoms. His complete blood count revealed a markedly elevated white count of 24.1. A differential was performed which revealed $66 \%$ segmented neutrophils and $3 \%$ bands. His erythrocyte sedimentation rate and $\mathrm{C}$ - reactive protein were both elevated at 84 and 3.646 respectively which suggested an inflammatory process rather than a physical injury. His white blood cell count was 24.1 , hemoglobin 9.9 , hematocrit 29.8 , and platelets 607.

Blood cultures were drawn which were pending at the time of diagnosis but showed no growth after 5 days. Plain radiographs were ordered of the ankle (Figure 1) (Figure 2), knee (Figure 3) (Figure 4), and tibia/fibula (Figure 5) (Figure 6) to which showed no evidence of fracture or malalignment. Arterial and venous ultrasounds of the lower extremities also did not show compromise of the tibial or dorsalis pedis artery or vein. Finally, nonvascular ultrasound of his lower extremity demonstrated a complex fluid collection within the left upper calf posteriorly, measuring $1.9 \times 2.7 \times 3.7 \mathrm{~cm}$ which appeared to involve the calf musculature (Figure 7).

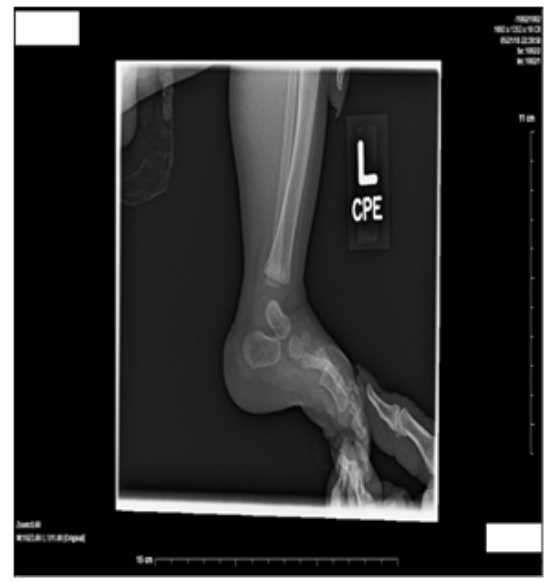

Figure I Plain radiograph of left ankle, lateral.

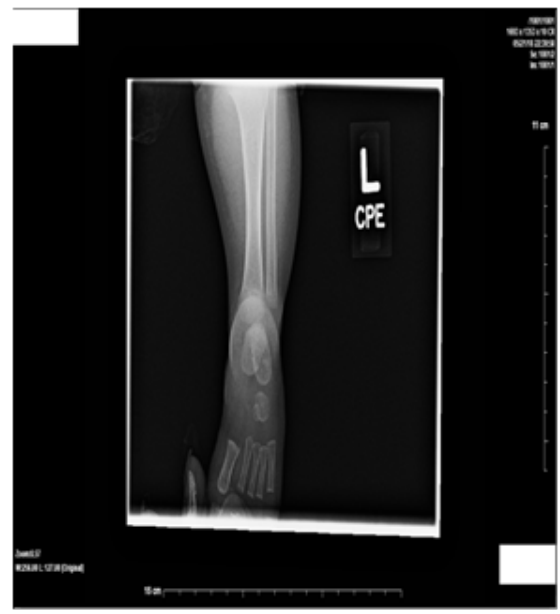

Figure 2 Plain radiograph of left ankle, posterior.

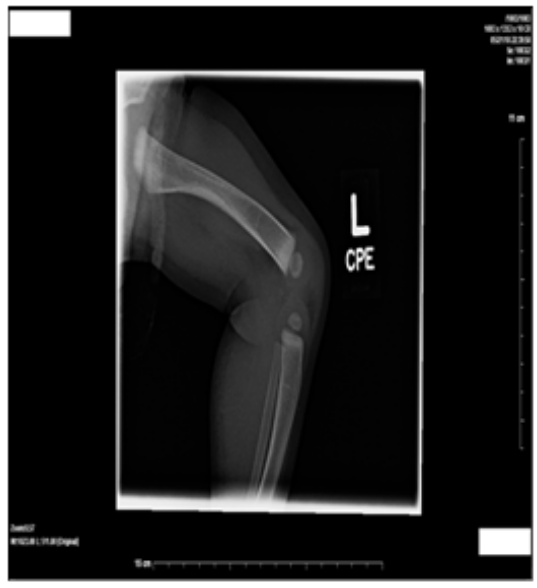

Figure 3 Plain radiograph of left knee, lateral. 


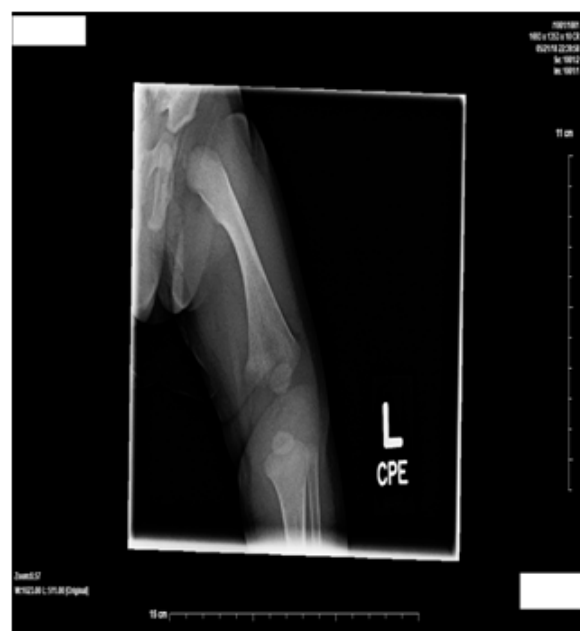

Figure 4 Plain radiograph of left knee.

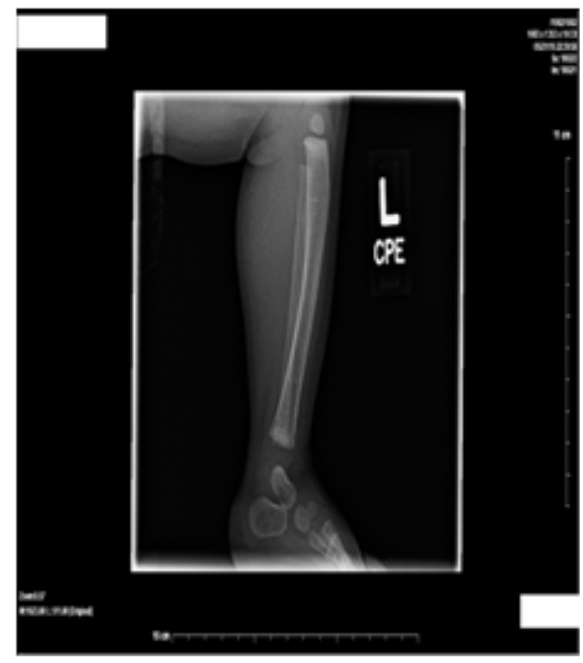

Figure 5 Plain radiograph of left tibia/fibula, lateral.

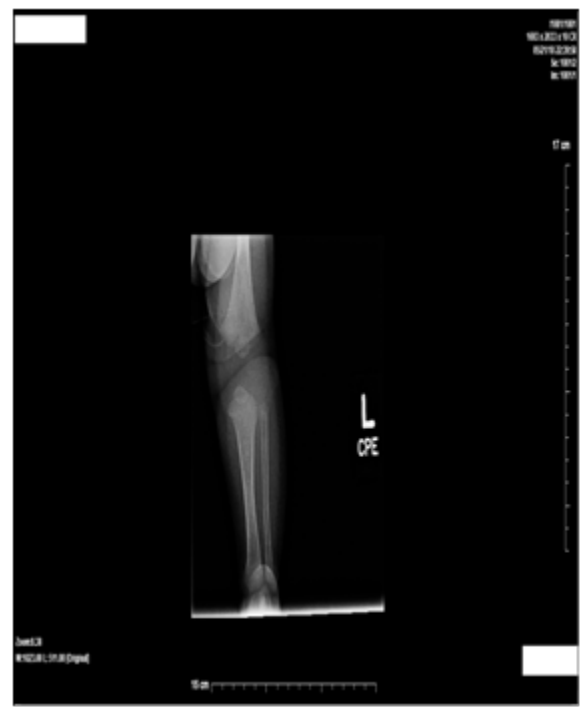

Figure 6 Plain radiograph of left tibia, fibula.

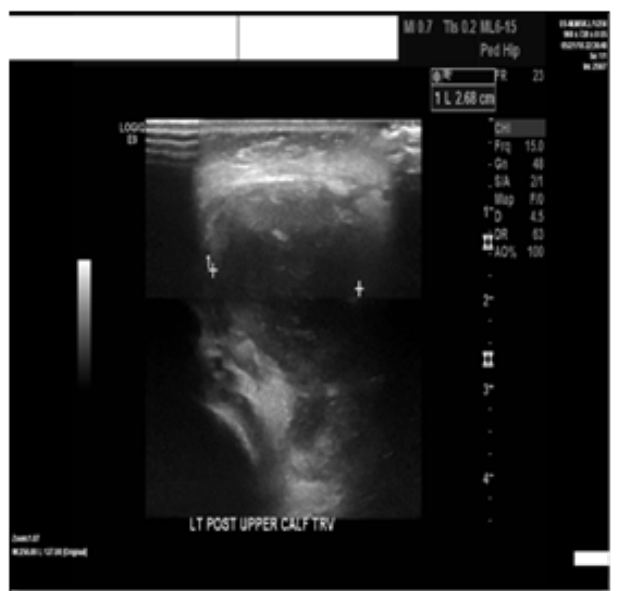

Figure 7 Ultrasound of posterior aspect of upper left calf, showing $1.9 \times 2.7$ $\times 3.7 \mathrm{~cm}$ abscess

\section{Discussion}

The combination of clinical presentation, imaging, and lab values suggested that our patient had a case of pyomyositis involving the gastronomies or soleus muscles. The treatment of pyomyositis is typically determined by the stage of disease. In stage 1 , the patient can present with campy local muscle pain, swelling, low-grade fever, and mild leukocytosis. An abscess or area of fluctuance is usually not obviously palpable. These patients can often be treated with IV antibiotics such as clindamycin and vancomycin. Stage 2 is characterized by higher fevers, more muscle tenderness, and edema of the extremity. An abscess is more commonly palpated in this stage and there will often be marked leukocytosis, which in our patient was considered to be a white count of over 18 . About $90 \%$ of patients present in this stage and would benefit from incision and drainage or needle aspiration of the abscess. They will often not have relief from antibiotics alone. Finally, stage 3 is the final stage of polymyositis and presents with signs of sepsis and systemic toxicity. If an individual is in stage 3, they will need more supportive management such as fluids in addition to antibiotics and surgery. ${ }^{6-8}$

Due to our patient's age, we opted for more conservative therapy with antibiotics first although he met certain criteria for stage 2. He was started on clindamycin which showed no improvement after one day and thus switched to vancomycin for broader spectrum coverage. On day 3 of admission, 8 days total since the onset of symptoms, it was decided to undergo incision and drainage of the posterior aspect of the upper left calf. Although he remained afebrile during the duration of his stay, he had no improvement in his physical exam and continued to have pain to passive movement of his lower extremity. A general orthopedic surgeon performed the procedure which resulted in expulsion of purulent, white fluid which was cultured. The incision was sealed and a penrose drain and ace bandage wrapping applied. On postoperative day 1, our patient's penrose drain was removed and he was found to still have significant swelling of his lower left extremity, but with improvement in his range of motion. Wound cultures were returned on the 7th day of his admission which showed moderate growth of methicillin-resistant Staphylococcus aureus. Susceptible antibiotics included clindamycin, rifampin, tetracycline, trimethoprim/sulfamethoxazole, and vancomycin. He was sent home 
with clindamycin to finish a course of 14 total days of antibiotics and scheduled for outpatient follow-up in one week. ${ }^{8}$

In our patient, it took 6 days from onset of symptoms to diagnosis which is consistent with the national average. His case was unique as he did not have any obvious predisposing factors towards the development of his pyomyositis and was also much younger than the typical patient, which also contributed to his delay in diagnosis and treatment. At first, his leg pain was assumed to be musculoskeletal in nature, and therefore was he was recommended pain relievers for pain. The differential for pyomyositis includes other skin and bone infections such as cellulitis, osteomyelitis, or joint disease such as septic arthritis. Another possibility could have been deep vein thrombosis due to his clinical presentation. Bone/muscle tumors could also not be ruled out. Lab results showed vague findings as elevated CRP, ESR, and WBC can be found in the majority of the mentioned pathology. Therefore, imaging including plain radiographs, MRI, and ultrasound were invaluable in facilitating proper diagnosis.

Antibiotic therapy should target gram-positive organisms, specifically staphylococci including methicillin-resistant Staphylococcus aureus. First-line therapy includes vancomycin or clindamycin for initial presentation and then adjusted according to susceptibilities found on culture. ${ }^{9}$ Antibiotics such as penicillin, although the drug of choice for most streptococcal infections, often are ineffective due to streptococcal organisms quickly reaching steady-state of growth in skeletal muscle. This is known as "the Eagle effect" and does not affect antibiotics such as clindamycin and vancomycin ${ }^{10}$ although the mainstay of treatment is usually surgical, it may be avoided if antibiotic therapy is begun earlier in the course of the disease.

Due to the uncertainty of etiology of pyomyositis in our patient, we discussed the possibility of an underlying immunodeficiency. There is also some underlying skepticism regarding other cases of pyomyositis due to the resilient nature of skeletal muscle. Blunt trauma is reported to occur in about $50 \%$ of cases studied by some authors, which was not present in our patient's case. ${ }^{11,12}$ Due to his age, our patient was also less likely to have undergone strenuous activity or exercise which would have caused microtrauma to his muscle. Pyomyositis has also been documented to be more common in temperate climates which are not consistent with the climate that our patient resides. ${ }^{13,14}$ finally, the involvement of the calf muscles, although possible, is not as common as involvement in the thigh and hip musculature. ${ }^{15}$ It is suggested that our patient's previous vaccinations may have served as a reservoir to growing methicillin-resistant Staphylococcus aureus, however, it is uncertain why his calf musculature became involved versus his thigh or hip.

\section{Conclusion}

Pyomyositis is a rare infection of skeletal muscle in children and can often present with vague symptoms and no obvious infection if the affected muscle is deeply situated. The nature of the disease calls for quick diagnosis and treatment due to the possibility of bacteremia and sepsis. Although more common in children ages 5-7 years old, there should be a high index of suspicion when a child demonstrates symptoms of pain localized to a large muscle group rather than bone or joint.

\section{Acknowledgements}

None.

\section{Conflict of interest}

Our authors did not report any potential conflicts of interests.

\section{References}

1. Stevens DL, Bisno AL, Chambers HF, et al. Practice guidelines for the diagnosis and management of skin and soft tissue infections: 2014 update by the infectious diseases society of America. Clin Infect Dis. 2014;15;59(2):e10-52.

2. Christin L, Sarosi GA. Pyomyositis in North America: case reports and review. Clin Infect Dis. 1992;15(4):668-77.

3. Gubbay AJ, Isaacs D. Pyomyositis in children. Pediatr Infect Dis J. 2000;19(10):1009-12.

4. Bickels J, Ben-Sira L, Kessler A, et al. Primary pyomyositis. J Bone Joint Surg. 2002;84-A(12):2277-86.

5. Gorwitz RJ. A review of community-associated methicillin-resistant Staphylococcus aureus skin and soft tissue infections. Pediatr Infect Dis J. 2008;27(1):1-7.

6. Gutierrez K. Bone and joint infections in children. Pediatr Clin North Am 2005;52(3):779-94.

7. Martínez AG, Avalos MA. Community-acquired, methicillin-resistant and methicillin-susceptible Staphylococcus aureus musculoskeletal infections in children. Pediatr Infect Dis J. 2004;23(8):701-6.

8. Mitsionis GI, Manoudis GN, Lykissas MG, et al. Pyomyositis in children: early diagnosis and treatment. J Pediatr Surg. 2009;44(11):2173-8.

9. Chiedozi LC. Pyomyositis, Review of 205 cases in 112 patients. Am J Surg. 1979;137(2):255-9.

10. Dennis L Stevens, Amy E Gibbons, Roberta Bergstrom, et al. The Eagle Effect Revisited: Efficacy of Clindamycin, Erythromycin, and Penicillin in the Treatment of Streptococcal Myositis. The Journal of Infectious Disease. 1988;158(1):23-28.

11. Renwick SE, Ritterbusch JF. Pyomyositis in children. J Pediatr Ortho. 1993;44(11):2173-8.

12. Jayoussi R, Bialik V, Eyal A, et al. Case report: pyomyositis caused by vigorous exercise in a boy. Acta Pediatr. 1995;84(2):226-7.

13. Chauhan S, Jain S, Varma S, et al. Tropical pyomyositis (myositis tropicans), current perspective. Postgrad Med J. 2004;80(943):267-70.

14. Comegna, Laura, et al. Pyomyositis Is Not Only a Tropical Pathology, a Case Series. Journal of Medical Case Reports. 2016;10(1):1-6.

15. Falasca GF, Reginato AJ. The spectrum of myositis and rhabdomyolysis associated with bacterial infection. J Rheumatol. 1994;21(10):1932-7. 